\title{
Signs for the onset of gluon saturation in exclusive photo-production of vector mesons
}

\author{
Martin Hentschinski* ${ }^{*}$ \\ Departamento de Actuaria, Física y Matemáticas Universidad de las Américas Puebla \\ Ex-Hacienda Santa Catarina Martir S/N, San Andrés Cholula 72820 Puebla, Mexico \\ E-mail: martin.hentschinski@udlap.mx
}

\section{Krzysztof Kutak}

The H. Niewodniczański Institute of Nuclear Physics, Polish Academy of Sciences, ul. Radzikowskiego 152, 31-342, Cracow, Poland

E-mail: krzysztof.kutakeifj.edu.pl

\begin{abstract}
We investigate the energy dependence of the photo-production cross-section of vector mesons $J / \Psi$ and $\Upsilon$, measured by both HERA experiments H1 and ZEUS in electron-proton collisions and by LHC experiments ALICE, CMS and LHCb in ultra-peripheral proton-proton and ultraperipheral proton-lead collisions. Our study uses 2 particular fits of inclusive unintegrated gluon distribution, based on non-linear Baltisky-Kovchegov evolution (Kutak-Sapeta gluon; KS) and next-to-leading order Baltisky-Fadin-Kuraev-Lipatov evolution (Hentschinski-Sabio Vera-Salas gluon; HSS). We find that linear next-to-leading order BFKL evolution can only describe production at highest energies, if perturbative corrections are increased to unnaturally large values; rendering this corrections small, the growth with energy is too strong in the LHC region and the description of $J / \Psi$ data fails. For the KS gluon we find that an accurate description of $J / \Psi$ data is possible if non-linear corrections to low x QCD evolution are taken into account; without such correction a description of data fails. We interpret this observation as a clear signal for the presence of high gluon densities in low $\mathrm{x}$ the proton, characteristic for the onset of gluon saturation.
\end{abstract}

7th Annual Conference on Large Hadron Collider Physics - LHCP2019

20-25 May, 2019

Puebla, Mexico

\footnotetext{
* Speaker.

${ }^{\dagger}$ Collaboration with Alfredo Arroyo Garcia is gratefully acknowledged.
} 


\section{Introduction}

The power-like rise of the gluon distribution at small $x$, with $x=M^{2} / s$ the ratio of the hard scale $M$ and the center of mass energy $\sqrt{s}$, is well established both experimentally [1] and theoretically $[2,3]$. Nevertheless such a power-like rise cannot continue forever. Bounds imposed by unitarity require that the observed rise in $x$ will eventually slow down and come to hold. From a theory point of view one expects the formation of an over occupied system of gluons, which eventually leads to saturation of gluon densities [4]; finding convincing phenomenological evidence for gluon saturation is still one of the open problems of Quantum Chromodynamics (QCD). The evolution from the low to large gluon densities is described by a set of nonlinear evolution equations, known as Balitsky-Jalilian-Marian-Iancu-McLerran-Weigert-Leonidov-Kovner; its frequently used mean field version is given by the Balitsky Kovchegov (BK) [5] evolution equation. A suitable process to study the low $x$ gluon at the Large Hadron Collider is provided by exclusive photo-production of vector mesons. For this observable a large amount of data has been collected both for the production of $J / \Psi$ and $\Upsilon$ vector mesons. The hard scale is in both cases provided by the heavy quark mass, i.e. the charm $(J / \Psi)$ and bottom ( $\Upsilon$ ) quark mass. In the case of the $J / \Psi$ one is therefore able to reach very small $x$ values at a low transverse scale, which allows for the potential observation of saturation effects. Photo-production of the $\Upsilon$ provides on the other hand a cross-check of the description well in the perturbative domain, where such effects are genuinely expected to be absent.

\section{Methodology}

To establish the presence of effects related to the presence of high gluon densities and the onset of gluon saturation, it is necessary but not sufficient to establish that frameworks which include corrections due to gluon saturation are capable to describe data. Instead it is necessary to establish the break-down of frameworks which do not include such effects. While the established description of QCD cross-sections in the perturbative low-density regime is based on collinear factorization and DGLAP evolution, we argue that it does not provide suitable benchmark for this particular observable: the $x$-dependence of collinear parton distribution functions is obtained from a fit to data at an initial hard scale which is usually of the order of the charm mass. As a consequence no perturbative evolution takes place for the description of $J / \Psi$ data. The description of the $x$ dependence is therefore a pure fit. The perturbative QCD enters only through DGLAP evolution, i.e. through the evolution from $J / \Psi$ to the $\Upsilon$ scale. For such evolution the following observations apply: a) $\Upsilon$ data are placed at large $x$ values than corresponding $J / \Psi$ data b) DGLAP evolution shifts large $x$ input to lower $x$ i.e. the low $x \Upsilon$ depends on the fit to $J / \Psi$ data at intermediate $x$, where absence of saturation effects is firmly established c) higher twist effects associated with high gluon densities die away fast within collinear DGLAP evolution. It is therefore far more useful to compare to linear Balitsky-Fadin-Kuraev-Lipatov (BFKL) low $x$ evolution instead: Perturbative QCD is being used to determine the low $x$ behavior of the gluon, based on fits of an unintegrated gluon distribution at intermediate values of $x$. A breakdown of such an description and the simultaneous description of data by a framework which incorporates high density effects, constitutes then a sign for the presence of effects related to gluon saturation. For a detailed discussion we refer the interested reader to [6], see also [7]. 


\section{Results}

In the following we will compare photo-production data to two particular QCD fits to combined HERA data which are subject to QCD low $x$ evolution. As the perturbative benchmark we will use the Hentschinski-Salas-Sabio Vera (HSS) [3] unintegrated gluon which is based on linear NLO BFKL evolution. To assess the importance of non-linear terms in low $x$ evolution equations, we use a particular solution to BK-evolution, with initial conditions fitted to combined HERA data by Kutak-Sapeta (KS) [8]. Both HSS and KS gluon include sub-leading terms related to the resummation of collinear logarithms. Our results are shown in Fig. 1. The results of our study for the fixed renormalization scales (i.e. an external scale related to the heavy quark mass) can be found in Fig. 1: continuous, black lines correspond to the KS-gluon and dotted, green lines to the HSSgluon at fixed scales. At first sight it appears that both the HSS (linear) and the KS (non-linear) gluon describe the energy dependence of the data. One might therefore conclude that there is no need for non-linear evolution associated with the presence of high gluon densities and that saturation effects are absent. Taking however a closer look at the dipole cross-section associated with the HSS-gluon one realizes that it takes the following form (see [6] for a detailed discussion). With the dipole cross-section $\sigma_{q \bar{q}}\left(x, r\right.$ obtained from the unintegrated gluon density $\mathscr{F}\left(x, \boldsymbol{k}^{2}\right)$ through $\sigma_{q \bar{q}}(x, r)=\frac{4 \pi}{N_{c}} \int \frac{d^{2} k}{k^{2}}\left(1-e^{i k \cdot r}\right) \alpha_{s} \mathscr{F}\left(x, \boldsymbol{k}^{2}\right)$, one has

$$
\sigma_{q \bar{q}}^{(\mathrm{HSS})}(x, r)=\alpha_{S} \hat{\sigma}_{q \bar{q}}^{(\mathrm{HSS})}(x, r), \quad \hat{\sigma}_{q \bar{q}}^{(\mathrm{HSS})}(x, r)=\hat{\sigma}_{q \bar{q}}^{(\mathrm{dom} .)}(x, r)+\hat{\sigma}_{q \bar{q}}^{(\text {corr. })}(x, r),
$$

where

$$
\begin{aligned}
& \hat{\sigma}_{q \bar{q}}^{\text {(dom) }}\left(x, r, M^{2}\right)=\int_{\frac{1}{2}-i \infty}^{\frac{1}{2}+i \infty} \frac{d \gamma}{2 \pi i}\left(\frac{4}{r^{2} Q_{0}^{2}}\right)^{\gamma} \frac{\bar{\alpha}_{s}\left(M \cdot Q_{0}\right)}{\bar{\alpha}_{s}\left(M^{2}\right)} f\left(\gamma, Q_{0}, \delta, r\right)\left(\frac{1}{x}\right)^{\chi\left(\gamma, M^{2}\right)} \\
& \hat{\sigma}_{q \bar{q}}^{\text {(corr. })}\left(x, r, M^{2}\right)=\int_{\frac{1}{2}-i \infty}^{\frac{1}{2}+i \infty} \frac{d \gamma}{2 \pi i}\left(\frac{4}{r^{2} Q_{0}^{2}}\right)^{\gamma} \frac{\bar{\alpha}_{s}\left(M \cdot Q_{0}\right)}{\bar{\alpha}_{s}\left(M^{2}\right)} f\left(\gamma, Q_{0}, \delta, r\right)\left(\frac{1}{x}\right)^{\chi\left(\gamma, M^{2}\right)} \\
& \times \frac{\bar{\alpha}_{s}^{2} \beta_{0} \chi_{0}(\gamma)}{8 N_{c}} \log \left(\frac{1}{x}\right)\left[-\psi(\delta-\gamma)+\log \frac{M^{2} r^{2}}{4}-\frac{1}{1-\gamma}-\psi(2-\gamma)-\psi(\gamma)\right],
\end{aligned}
$$

The second term constitutes a perturbative correction for the next-to-leading logarithmic resummation, since it is of order $\alpha^{n+1} \ln ^{n}(1 / x), n \geq 0$. Even though this term is formally sub-leading, it grows with decreasing $x$ and the logarithm can eventually compensate smallness of the perturbative strong coupling $\alpha_{s}$. This is behavior is explored in Fig. 2. We find that if we set the hard-scale to a fixed value $M^{2}=3.27 \mathrm{GeV}^{2}$, the perturbative correction is indeed small for $x$ values typical for HERA kinematics, for which the original fit has been performed. Turning on the other hand to the smallest $x$ values probed in photo-production of $J / \Psi \mathrm{s}$ at the LHC, the corrections supersedes the formally leading term; the perturbative expansion is breaking down. There is an easy fix to this problem, namely choosing an $r$-dependent renormalization scale. This removes the $r$-dependent logarithm in the correction term and consequently reduces the size of the correction in the critical region. Having stabilized the linear NLO BFKL evolution, we return to data: while the new solution (dashed green line) essential agrees with the fixed scale solution for the $\Upsilon$ and for the $J / \Psi$ 

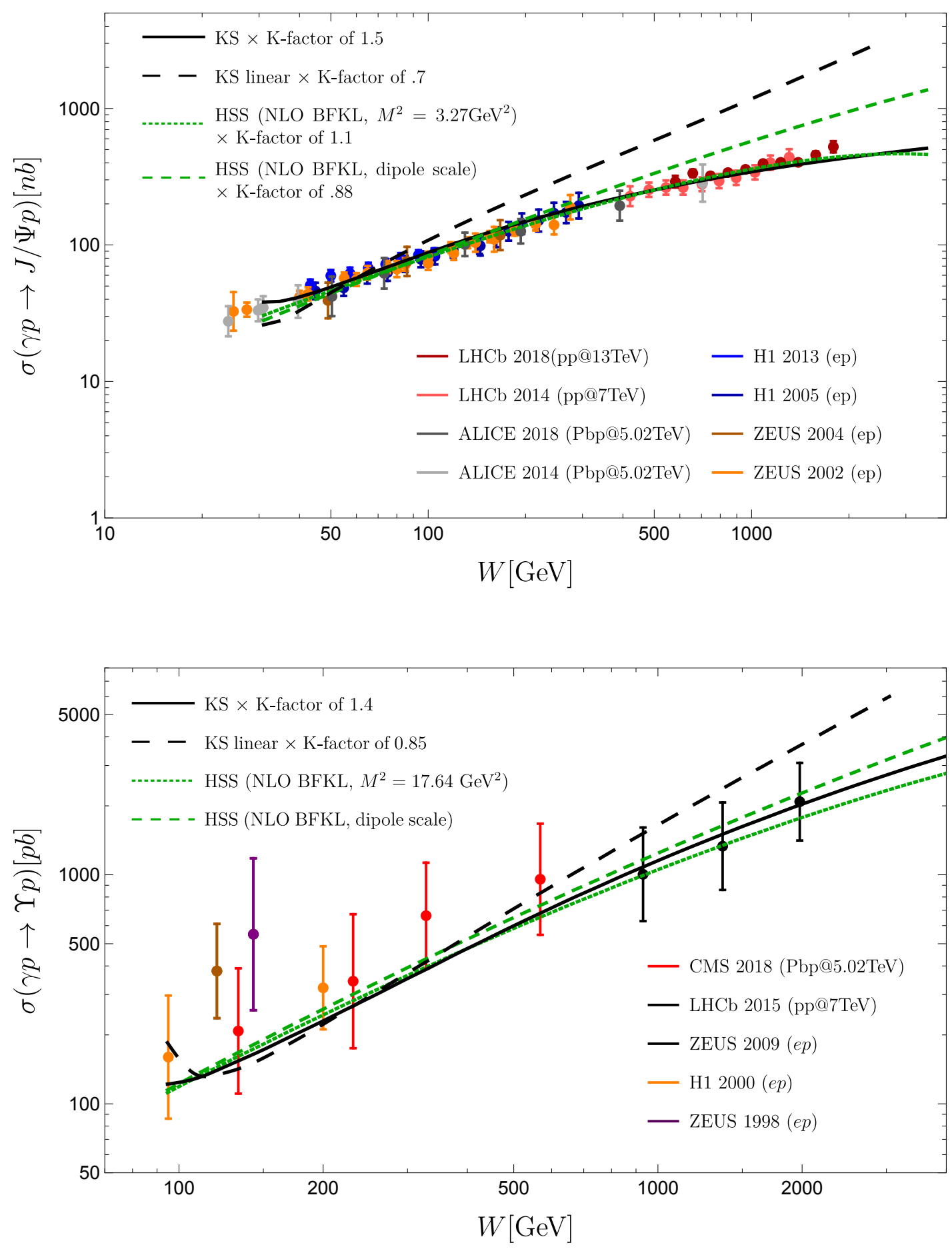

Figure 1: Energy dependence of the $J / \Psi$ and $\Upsilon$ photo-production cross-section as provided by the KS and HSS gluon distribution. The HSS distribution with dipole size scale corresponds to a specific scale setting for the HSS gluon discussed in Sec. 3. For the $J / \Psi$ we further display photo-production data measured at HERA by ZEUS and HI collaborations [9] as well as LHC data obtained from ALICE and LHCb [10]. For the $\Upsilon$ cross-section we show HERA data measured by HI and ZEUS [11] and LHC data by LHCb and CMS [12]. 


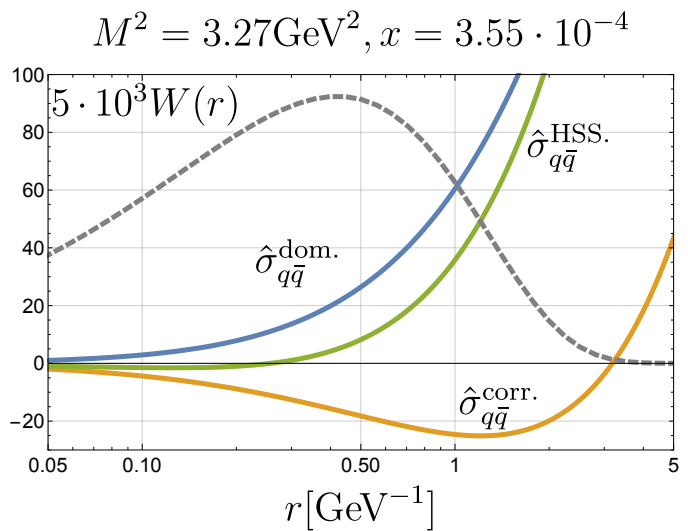

(a)

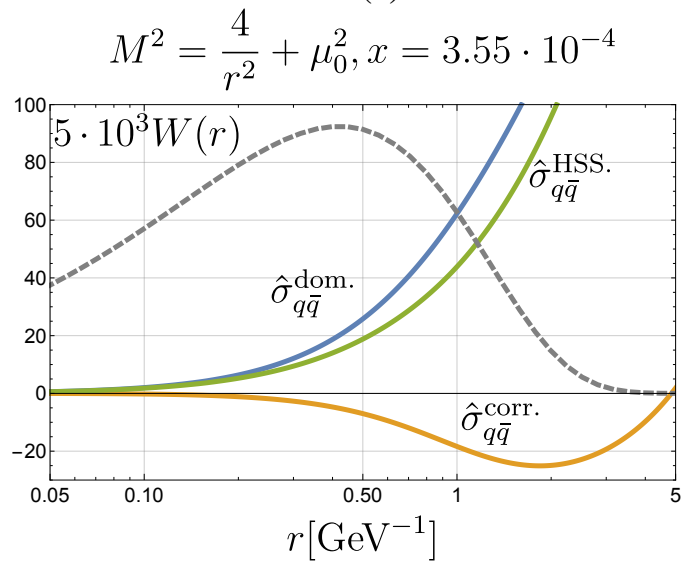

(c)

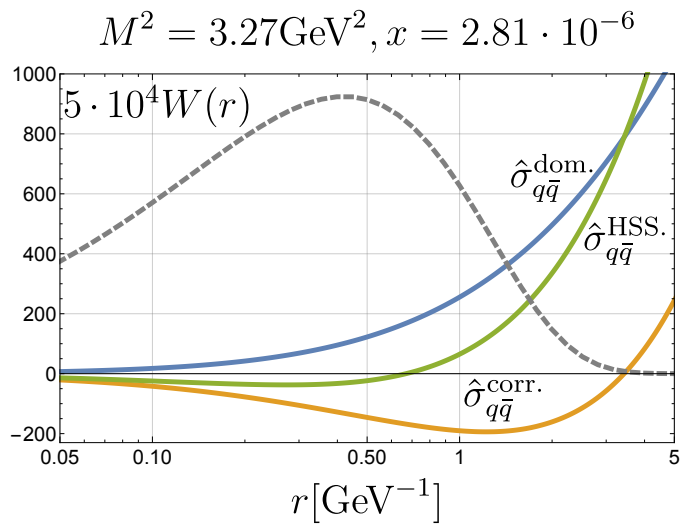

(b)

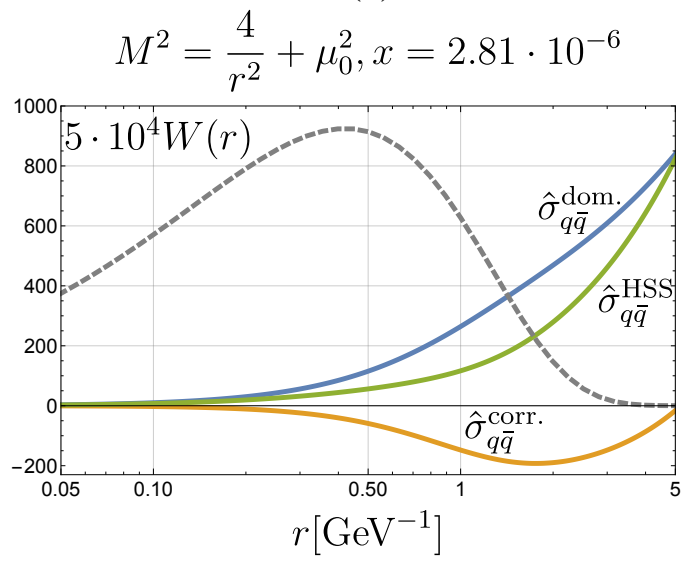

(d)

Figure 2: HSS dipole cross-section (with an overall factor of $\alpha_{s}$ extracted) at fixed $(J / \Psi$-scale, top row) and $r^{2}$-dependent scale (bottom row) in units of $\mathrm{GeV}^{-2}$. The function $W(r)$ indicates the typical dipole sized probed in $J / \Psi$ photo-production.

in the HERA region (the observed deviation is of the order of a typical variation of the renormalization scale, see [7]), the stabilized linear NLO BFKL evolution overshoots data for the $J / \Psi$ in the LHC region. At the same time the energy dependence of the data is very well described by the non-linear KS gluon. To assess the importance of the non-linearities in the solution, we also compare to the KS-gluon with non-linearities turned off (dashed black line): We observe that the linear KS-gluon overshoots data. We therefore conclude that non-linear effects are essential to describe the energy dependence of $J / \Psi$ data in the LHC region. We interpret this as a clear sign for the onset of saturation effects in this region of phase space.

\section{Conclusions}

The observed slow-down of the growth with energy is one of the core predictions of gluon saturation. To establish the observation made in this letter it is therefore necessary to search for different observables which probe the low $x$ gluon in a similar kinematic regime and to increase fur- 
ther the theoretical accuracy of the underlying framework. Steps to address the latter are currently undertaken in [13] (azimuthal de-correlations of dijets) and [14,15] (theory developments).

\section{References}

[1] H. Abramowicz et al. [H1 and ZEUS Collaborations], Eur. Phys. J. C 75, no. 12, 580 (2015) doi:10.1140/epjc/s10052-015-3710-4 [arXiv:1506.06042 [hep-ex]].

[2] L. N. Lipatov, Sov. J. Nucl. Phys. 23 (1976) 338, E. A. Kuraev, L. N. Lipatov, V. S. Fadin, Phys. Lett. B 60 (1975) 50, Sov. Phys. JETP 44 (1976) 443, Sov. Phys. JETP 45 (1977) 199. I. I. Balitsky, L. N. Lipatov, Sov. J. Nucl. Phys. 28 (1978) 822.

[3] M. Hentschinski, A. Sabio Vera and C. Salas, Phys. Rev. Lett. 110 (2013) no.4, 041601 [arXiv:1209.1353 [hep-ph]].

[4] L. V. Gribov, E. M. Levin and M. G. Ryskin, Phys. Rept. 100 (1983) 1. doi:10.1016/0370-1573(83)90022-4

[5] I. Balitsky, Nucl. Phys. B 463 (1996) 99 doi:10.1016/0550-3213(95)00638-9 [hep-ph/9509348]; Y. V. Kovchegov, Phys. Rev. D 60 (1999) 034008 doi:10.1103/PhysRevD.60.034008 [hep-ph/9901281].

[6] A. Arroyo Garcia, M. Hentschinski and K. Kutak, Phys. Lett. B 795, 569 (2019) doi:10.1016/j.physletb.2019.06.061 [arXiv:1904.04394 [hep-ph]].

[7] I. Bautista, A. Fernandez Tellez and M. Hentschinski, Phys. Rev. D 94, no. 5, 054002 (2016) doi:10.1103/PhysRevD.94.054002 [arXiv:1607.05203 [hep-ph]].

[8] K. Kutak and S. Sapeta, Phys. Rev. D 86 (2012) 094043 doi:10.1103/PhysRevD.86.094043 [arXiv:1205.5035 [hep-ph]].

[9] S. Chekanov et al. [ZEUS Collaboration], Eur. Phys. J. C 24, 345 (2002) [hep-ex/0201043]; S. Chekanov et al. [ZEUS Collaboration], Nucl. Phys. B 695, 3 (2004) [hep-ex/0404008]; C. Alexa et al. [H1 Collaboration], Eur. Phys. J. C 73, no. 6, 2466 (2013) [arXiv:1304.5162 [hep-ex]]; A. Aktas et al. [H1 Collaboration], Eur. Phys. J. C 46, 585 (2006) [hep-ex/0510016].

[10] B. B. Abelev et al. [ALICE Collaboration], Phys. Rev. Lett. 113, no. 23, 232504 (2014) [arXiv:1406.7819 [nucl-ex]]; S. Acharya et al. [ALICE Collaboration], Eur. Phys. J. C 79, no. 5, 402 (2019) doi:10.1140/epjc/s10052-019-6816-2 [arXiv:1809.03235 [nucl-ex]];

R. Aaij et al. [LHCb Collaboration], J. Phys. G 40, 045001 (2013) [arXiv:1301.7084 [hep-ex]]; J. Phys. G 41, 055002 (2014) [arXiv:1401.3288 [hep-ex]]; R. Aaij et al. [LHCb Collaboration], JHEP 1810, 167 (2018) doi:10.1007/JHEP10(2018)167 [arXiv:1806.04079 [hep-ex]].

[11] C. Adloff et al. [H1 Collaboration], Phys. Lett. B 483, 23 (2000) [hep-ex/0003020]; J. Breitweg et al. [ZEUS Collaboration], Phys. Lett. B 437 (1998) 432 [hep-ex/9807020]; S. Chekanov et al. [ZEUS Collaboration], Phys. Lett. B 680, 4 (2009) [arXiv:0903.4205 [hep-ex]].

[12] R. Aaij et al. [LHCb Collaboration], JHEP 1509, 084 (2015) [arXiv:1505.08139 [hep-ex]]; CMS Collaboration [CMS Collaboration], "Measurement of exclusive $\mathrm{Y}$ photoproduction in $\mathrm{pPb}$ collisions at $\sqrt{s_{\mathrm{NN}}}=5.02 \mathrm{TeV}$," CMS-PAS-FSQ-13-009; A. M. Sirunyan et al. [CMS Collaboration], Eur. Phys. J. C 79, no. 3, 277 (2019) doi:10.1140/epjc/s10052-019-6774-8 [arXiv:1809.11080 [hep-ex]].

[13] A. van Hameren, P. Kotko, K. Kutak and S. Sapeta, arXiv:1903.01361 [hep-ph]. 
[14] M. Hentschinski, A. Kusina, K. Kutak and M. Serino, Eur. Phys. J. C 78 (2018) no.3, 174 doi:10.1140/epjc/s10052-018-5634-2 [arXiv:1711.04587 [hep-ph]]. M. Hentschinski, A. Kusina and K. Kutak, Phys. Rev. D 94 (2016) no.11, 114013 doi:10.1103/PhysRevD.94.114013 [arXiv:1607.01507 [hep-ph]]. O. Gituliar, M. Hentschinski and K. Kutak, JHEP 1601 (2016) 181 doi:10.1007/JHEP01(2016)181 [arXiv:1511.08439 [hep-ph]].

[15] M. Hentschinski, Phys. Rev. D 97 (2018) no.11, 114027 doi:10.1103/PhysRevD.97.114027 [arXiv:1802.06755 [hep-ph]]. M. Hentschinski and A. Sabio Vera, Phys. Rev. D 85 (2012) 056006 [arXiv:1110.6741 [hep-ph]]; M. Hentschinski, Nucl. Phys. B 859 (2012) 129 [arXiv:1112.4509 [hep-ph]]; G. Chachamis, M. Hentschinski, J. D. Madrigal Martinez and A. Sabio Vera, Nucl. Phys. B 876 (2013) 453 [arXiv:1307.2591 [hep-ph]]; Phys. Rev. D 87 (2013) no.7, 076009 [arXiv:1212.4992]; Nucl. Phys. B 861 (2012) 133 [arXiv:1202.0649 [hep-ph]]; M. Hentschinski, J. D. Madrigal Martínez, B. Murdaca and A. Sabio Vera, Phys. Lett. B 735 (2014) 168 [arXiv:1404.2937 [hep-ph]]; Nucl. Phys. B 887 (2014) 309 [arXiv:1406.5625 [hep-ph]]; Nucl. Phys. B 889 (2014) 549 [arXiv:1409.6704 [hep-ph]]; 